\title{
Incidence and risk factors of readmission after esophagectomy for esophageal cancer
}

\author{
Seong Yong Park, Dae Joon Kim, Go Eun Byun \\ Department of Thoracic and Cardiovascular Surgery, Yonsei University College of Medicine, Seoul, Republic of Korea \\ Contributions: (I) Conception and design: SY Park; (II) Administrative support: SY Park; (III) Assessment of study materials or patients: All authors; (IV) \\ Collection and assembly of data: SY Park; GE Byun; (V) Data analysis and interpretation: SY Park; DJ Kim; (VI) Manuscript writing: All authors; (VII) \\ Final approval of manuscript: All authors. \\ Correspondence to: Dae Joon Kim, MD, PhD. Department of Thoracic and Cardiovascular Surgery, Yonsei University College of Medicine, 50 Yonsei- \\ ro, Seodaemun-gu, Seoul, 120-752, Republic of Korea. Email: kdjcool@yuhs.ac.
}

\begin{abstract}
Background: The Esophageal Complications Consensus Group (ECCG) recommends that readmission to a primary or secondary hospital within 30 days of discharge after esophagectomy is an important quality outcome indicator for esophagectomy. This retrospective study was performed to investigate the incidence and risk factors for readmission after esophagectomy.
\end{abstract}

Methods: We retrospectively reviewed 291 patients who received an esophagectomy and mediastinal lymphadenectomy for curative purposes from January 2006 to June 2017.

Results: The mean age was $63.02 \pm 8.02$ years, and there were $264(90.7 \%)$ male patients. Thirty-nine (13.4\%) patients were re-admitted within 30 days after discharge. The mean interval from discharge to the readmission was $13.46 \pm 9.36$. Common causes of readmission were anastomotic stricture that required ballooning (12, 30.7\%), wound problem (7, 17.9\%), pneumonia (6, 15.4\%), and poor oral intake (4, 10.2\%). Other causes of readmission were delayed gastric emptying [3], jejunostomy tube problem [2], ileus [2], pain [1], pneumothorax [1], and pleural effusion [1]. On multivariable analysis, anastomotic leakage (odd ratio $=2.884, \mathrm{P}=0.026$ ) was significantly related to readmission, whereas age, pathologic stage, vocal cord palsy, and neoadjuvant therapy were not related to readmission.

Conclusions: Readmission within 30 days after esophagectomy was determined to be related to postoperative anastomotic leakage and wound problems whereas the vocal cord palsy was not.

Keywords: Esophagus; readmission; esophagectomy; operative outcomes

Submitted Jul 04, 2019. Accepted for publication Oct 08, 2019.

doi: $10.21037 /$ jtd.2019.10.34

View this article at: http://dx.doi.org/10.21037/jtd.2019.10.34

\section{Introduction}

Esophagectomy and mediastinal lymph node dissection are a principal treatment not only for early esophageal cancer, but also for locally advanced esophageal cancer as a vital component of multimodal therapy. However, esophagectomy for esophageal cancer is a high-risk surgical procedure, and in spite of the advancements in operative techniques and perioperative care approaches, esophagectomy can be associated with early postoperative mortality ranging from $1 \%$ to $13 \%$ of cases (1). Overall postoperative morbidity rates have been reported to be up to $50 \%$ (2), and 30-day readmission rates have been reported at $12 \%$ to $25 \%$, which is higher than those for most other oncologic surgeries (3). These readmissions can increase unnecessary medical cost; in addition, readmission after esophagectomy for esophageal cancer has been reported to be related to poor survival regardless of initial stage and treatments (4).

The Esophageal Complications Consensus Group 
(ECCG) suggested that readmission to a primary or secondary hospital within 30 days of discharge after esophagectomy can be an important quality outcome indicator for esophagectomy (5). However, few research reports have analyzed the incidence of readmission after esophagectomy, particularly with a focus on risk factors that predispose patients with esophageal cancer to readmission after esophagectomy (3). To improve the quality of operative outcomes and patient care and to reduce unnecessary medical cost, esophageal surgeons must be aware of the incidence and risk factors of readmission after esophagectomy. Therefore, this retrospective study investigated the incidence and risk factors of readmission after elective esophagectomy for esophageal cancer based on data from a single institution.

\section{Methods}

This retrospective study was approved by the institutional review board of our hospital (4-2018-0580). Records of 291 patients who underwent elective esophagectomy with mediastinal and abdominal lymphadenectomy for esophageal cancer in the tertiary academic institution, from January 2006 to June 2017, were retrospectively reviewed. Salvage or palliative operation and operative mortality were used as exclusion criteria. The routine surgical procedures were McKeown esophagectomy; esophagectomy and total lymphadenectomy and cervical esophagogastrostomy. The minimally invasive approaches by robot or thoracoscopy were mainly applied in the thoracic phase, the laparotomy was usually done for the abdominal phase. The pyloric drainage procedures were not performed and jejunostomy tube was placed routinely. Even though we did not routinely apply the enhanced recovery after surgery (ERAS) program, the jejunostomy feeding was started at postoperative day (POD) 3 and the esophagography and vocal cord evaluation was done at POD 7 after operation. Sips of water and oral feeding were usually started at POD 7 . The antithrombotic agents were not used at the immediate postoperative periods.

Patient factors, past medical history, and surgical and postoperative stay information were acquired from the institutional medical database system. Patient factors were age, sex, tumor location, histology, pathologic stage, and comorbidities. As a comorbidity, pulmonary disease was defined as the history of chronic obstructive pulmonary disease, pulmonary tuberculosis, emphysema, or bronchial asthma. Cardiac disease was defined as history of hypertension, coronary artery obstructive disease, valve disease, or arrhythmia. Postoperative factors were length of stay and postoperative complications. Pulmonary complications were defined as any pneumonic infiltrates on chest X-ray, acute lung injury, or adult respiratory distress syndrome (ARDS). Vocal cord examinations were routinely performed by an otolaryngologist to identify recurrent laryngeal nerve palsies. All patients underwent videostroboscopy to evaluate vocal fold motion and glottal configuration during inspiration and phonation at POD 7. Decreased vocal fold movement and fixed vocal folds were defined as vocal cord palsies. Esophagography was performed at POD 7 to rule out anastomotic leakage. Arrhythmia included all types of arrhythmias, such as atrial fibrillations or atrial flutters. Prolonged air leakage was defined as air leakage that was sustained beyond POD 7 . All data were managed by the dedicated data manager (GE Byun). The severity of postoperative complications was graded by Clavien-Dindo classification (6). Readmission within 30 days after discharge was defined as inpatient admission to our institution within 30 days of discharge from a postoperative stay. The causes of readmission were analyzed based on the medical records. The costs generated during the readmission periods were calculated based on the institutional medical system and it defined as total cost for readmission. The final pathologic esophageal cancer stage was classified based on the 8th edition of the American Joint Committee on Cancer system of esophageal cancer (7).

\section{Statistical analysis}

Statistical analysis was performed using the open source statistical software R (http://www.R-project.org). Clinical and pathological parameters were described as mean \pm standard deviation for continuous variables and frequency (\%) for categorical variables if the variables showed normal distribution. If variables did not show normal distribution, the parameters were described as median (inter-quantile range). Comparison of continuous variables was performed using Student's $t$-test (2-sided). Logistic regression was used to identify risk factors associated with 30-day readmission. Variables identified as significant in univariable models $(\mathrm{P}<0.2)$ were included as covariates in the multivariable model. Odds ratios (ORs) and corresponding 95\% confidence intervals (CIs) are reported. All tests were twosided, and the criterion for significance was $\mathrm{P}<0.05$. 
Table 1 Patient characteristics $(\mathrm{n}=291)$

\begin{tabular}{|c|c|}
\hline Variables & Value \\
\hline Age (years) & $63.02 \pm 8.02$ \\
\hline Male & $264(90.7)$ \\
\hline Smoking history (pack-year) & $33.76 \pm 18.27$ \\
\hline $\mathrm{FEV}_{1}(\%)$ & $99.90 \pm 18.27$ \\
\hline FVC (\%) & $97.81 \pm 17.87$ \\
\hline \multicolumn{2}{|l|}{ Charlson Comorbidity Index } \\
\hline 0 & $93(32.0)$ \\
\hline 1 & 97 (33.3) \\
\hline 2 & $53(18.2)$ \\
\hline 3 & $38(13.1)$ \\
\hline 4 & $10(3.4)$ \\
\hline Cardiac disease & $82(28.2)$ \\
\hline Diabetes mellitus & $34(11.7)$ \\
\hline Pulmonary disease & $52(17.9)$ \\
\hline \multicolumn{2}{|l|}{ Pathology } \\
\hline Squamous cell carcinoma & 279 (95.9) \\
\hline Adenocarcinoma & $12(4.1)$ \\
\hline \multicolumn{2}{|l|}{ Location } \\
\hline Upper & $24(8.2)$ \\
\hline Mid & $157(54.0)$ \\
\hline Lower & $110(37.8)$ \\
\hline \multicolumn{2}{|l|}{ Pathologic stage } \\
\hline 0 & $21(7.2)$ \\
\hline 1 & $112(38.5)$ \\
\hline II & $71(24.4)$ \\
\hline III & $74(25.4)$ \\
\hline IV & $13(4.5)$ \\
\hline Neoadjuvant therapy & $41(14.1)$ \\
\hline
\end{tabular}

The data were described as mean \pm standard deviation for continuous variables and frequency (\%). $\mathrm{FEV}_{1}$, forced expiratory volume in the first second; FVC, forced vital capacity.

\section{Results}

\section{Patient demographics and postoperative complications}

Basic patient characteristics are described in Table 1. The mean patient age was $63.02 \pm 8.02$ years, and $264(90.7 \%)$
Table 2 Postoperative outcomes $(\mathrm{n}=291)$

\begin{tabular}{lc}
\hline Variables & Value \\
\hline Postoperative complications, $\mathrm{n}(\%)$ & $62(21.3)$ \\
Vocal cord palsy & $35(12.0)$ \\
Pulmonary complications & $30(10.3)$ \\
Anastomotic leakage & $2(0.7)$ \\
Chyloperitoneum & $3(1.0)$ \\
Chylothorax & $3(1.0)$ \\
Wound problem & $2(0.7)$ \\
Postoperative bleeding & $1(0.3)$ \\
Prolonged air leakage & $10(3.43)$ \\
Pleural effusion & $3(1.0)$ \\
Acute renal failure & $7(2.4)$ \\
Arrhythmia & $1[1-3]$ \\
Intensive care unit stay (days), median [IQR] & $17[14-26]$ \\
Hospital stay (days), median [IQR]
\end{tabular}

patients were male. Squamous cell carcinoma was identified as the majority of the pathologies (95.9\%), and $14.1 \%$ of patients received neoadjuvant therapy.

The postoperative complications and outcomes are summarized in Table 2. These complications meant the complication which occurred during the first admission. In 134 patients showed postoperative complications. The highest grade based on the Clavien-Dindo classification was as follows; 19 in grade 1, 61 in grade 2, 31 in grade 3 and 23 in grade 4 , respectively. The most common complication was vocal cord palsy $(21.3 \%)$. The mean hospital stay was $25.61 \pm 27.42$ days. The hospital stays were significantly higher in patients with postoperative complications than in patients without postoperative complications $(16.0 \pm 6.10 \mathrm{vs}$. $34.93 \pm 35.68$ days, $\mathrm{P}<0.001)$.

\section{Incidence and reasons for readmission}

Thirty-nine (13.4\%) patients were re-admitted within 30 days after discharge. There were 24 (61.5\%) among the 39 patients readmitted within 2 weeks after initial discharge. The mean interval from initial discharge to readmission was $13.46 \pm 9.36$ days. Causes for readmission are summarized in Table 3. Common causes of readmission were anastomotic stricture requiring ballooning (12, 30.7\%), wound problem 
Table 3 Characteristics and causes of readmission ( $n=39)$

\begin{tabular}{|c|c|}
\hline Characteristics & Value \\
\hline Length of stay during readmission (days), median [IQR] & 8 [3-16] \\
\hline Total cost for readmission (Korean Wons), median [IQR] & $2,619,812[1,075,895-6,269,485]$ \\
\hline \multicolumn{2}{|l|}{ Cause of readmission, $\mathrm{n}(\%)$} \\
\hline Wound problem & $7(17.9)$ \\
\hline Pneumonia & $6(15.4)$ \\
\hline Poor oral intake & $4(10.2)$ \\
\hline Delayed gastric emptying & $3(7.7)$ \\
\hline Pain & $1(2.6)$ \\
\hline Pneumothorax & $1(2.6)$ \\
\hline Pleural effusion requiring closed drainage & $1(2.6)$ \\
\hline Numbers of patients who required the procedure during readmission & $15(38.4)$ \\
\hline
\end{tabular}

(7, 17.9\%), pneumonia (6, 15.4\%), and poor oral intake (4, $10.2 \%)$. Other causes of readmission were delayed gastric emptying [3], jejunostomy tube problem [2], ileus [2], pain [1], pneumothorax [1], and pleural effusion [1]. Among the 7 patients with wound problems, 4 showed neck wound problems, and the other 3 patients had wound problems at the chest tube site or abdomen. The mean hospital stay for readmission was $11.41 \pm 10.92$ days (range, $1-38$ days). The mean total cost for readmission was 4,386,278 $44,492,302$ Korean won (approximately \$4,041 US dollars).

\section{Risk factors of readmission}

On univariable analysis, history of pulmonary disease ( $\mathrm{OR}=2.132$, 95\% CI: 1.159-3.920, $\mathrm{P}=0.015)$, vocal cord palsy $(\mathrm{OR}=2.758,95 \% \mathrm{CI}: 1.554-4.896, \mathrm{P}=0.001)$, and anastomotic leakage $(\mathrm{OR}=2.247,95 \% \mathrm{CI}: 1.049-4.813$, $\mathrm{P}=0.037)$ were primary risk factors of readmission after esophagectomy. Frequency of hospital stay was significantly higher in patients with postoperative complications than in patients without postoperative complications $(\mathrm{P}<0.001)$; the length of hospital stay was not included in multivariable analysis. On multivariable analysis, only postoperative anastomotic leakage was identified as a risk factor of readmission after esophagectomy $(\mathrm{OR}=2.884$, 95\% CI: $1.133-7.343, \mathrm{P}=0.026)$, whereas vocal cord palsy and pathologic stages were not related to readmission. In 30 patients with postoperative anastomotic leakage, the frequency of readmission due to wound problem $(13.3 \% \mathrm{vs}$. $1.1 \%, \mathrm{P}=0.003)$ or anastomotic stricture $(13.3 \%$ vs. $3.4 \%$, $\mathrm{P}=0.034)$ was significantly higher than patients without anastomotic leakage (Table 4).

\section{Discussion}

Our data showed that the rate of readmission within 30 days after esophagectomy was $13.4 \%$ and was primarily related to postoperative anastomotic leakage and wound problem. Readmission resulted in additional hospital cost of 4,386,278 $\pm 4,492,302$ Korean won (approximately $\$ 4,041$ US dollars). The readmission rate and risk factors reported in previous studies are summarized in Table 5. Previous studies showed similar incidence of readmission after esophagectomy to our results, about 12.6-25\% $(1,4)$. Previous studies have proposed possible risk factors for readmission, including postoperative complications, 
Table 4 Risk factors of readmission after esophagectomy

\begin{tabular}{|c|c|c|c|c|}
\hline Variables & \multicolumn{2}{|c|}{ Univariable analysis } & \multicolumn{2}{|c|}{ Multivariable analysis } \\
\hline Sex & $1.660(0.667-4.067)$ & 0.268 & - & - \\
\hline Age & $1.004(0.975-1.034)$ & 0.797 & - & - \\
\hline \multicolumn{5}{|l|}{ Charlson comorbidity index } \\
\hline 3-4 (vs. 0) & $1.420(0.559-3.607)$ & 0.461 & - & - \\
\hline Neoadjuvant therapy & $0.666(0.324-1.369)$ & 0.269 & - & - \\
\hline \multicolumn{5}{|l|}{ Pathologic stage } \\
\hline I (vs. 0) & $1.219(0.413-3.603)$ & 0.072 & $1.855(0.378-9.095)$ & 0.446 \\
\hline Cardiac disease & $1.870(1.106-3.161)$ & 0.019 & $1.597(0.766-3.330)$ & 0.212 \\
\hline Diabetes mellitus & $1.061(0.507-2.221)$ & 0.875 & - & - \\
\hline Pulmonary disease & $2.132(1.159-3.920)$ & 0.015 & $1.708(0.753-3.877)$ & 0.200 \\
\hline \multicolumn{5}{|l|}{ Postoperative complications } \\
\hline Vocal cord palsy & $2.758(1.554-4.896)$ & 0.001 & $1.241(0.560-2.749)$ & 0.596 \\
\hline Pulmonary complications & $0.930(0.442-1.956)$ & 0.848 & - & - \\
\hline Anastomotic leakage & 2.247 (1.049-4.813) & 0.037 & $2.884(1.133-7.343)$ & 0.026 \\
\hline
\end{tabular}

Table 5 Summary of the literature on readmission after esophagectomy

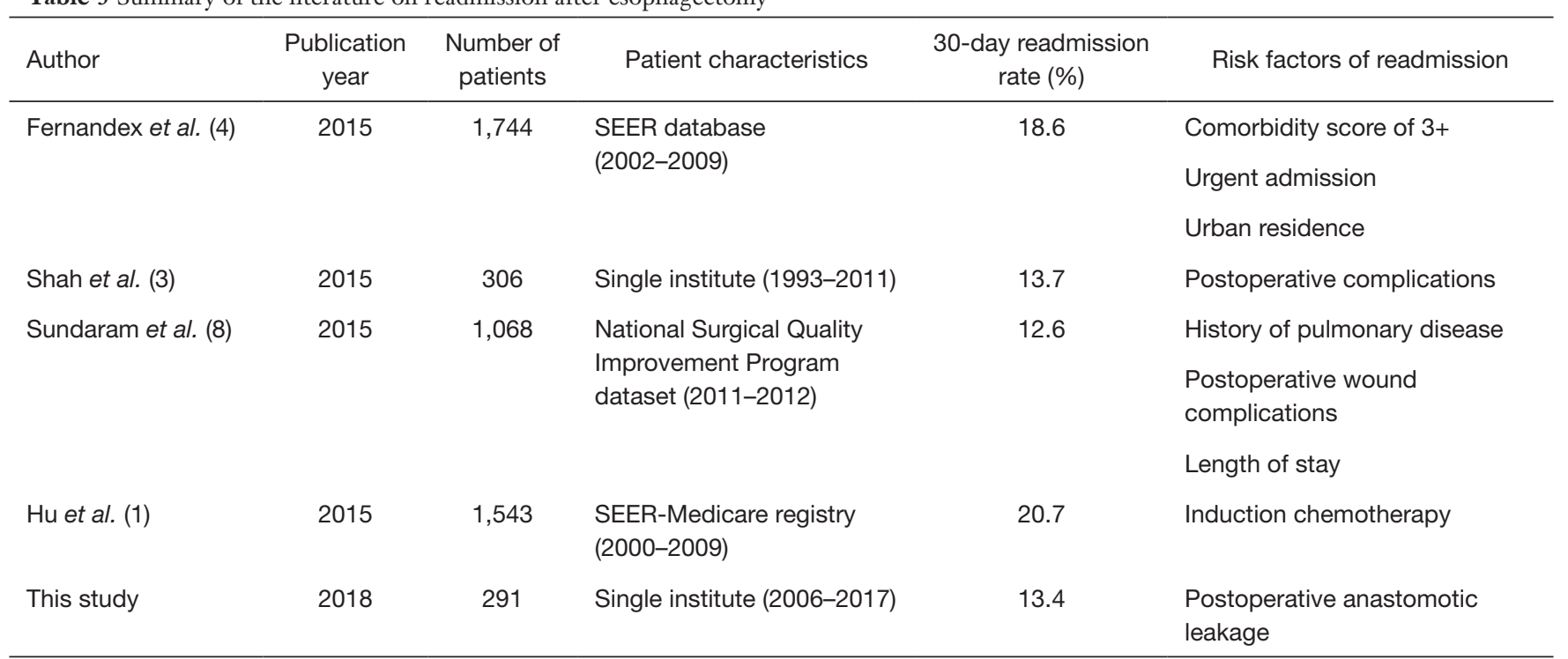

SEER, Surveillance, Epidemiology, and End Results Program. 
thromboembolic events, pulmonary complications, wound problems, and longer hospital stay $(3,4,8)$. The initial hospital stay was not included in this risk factor analysis because we thought that prolonged hospital stay reflects the postoperative complications. A hospital stay is naturally prolonged if postoperative complications occur. Shah et al. reported that postoperative complications were related to readmission after esophagectomy, and that complications that were not adequately managed before discharge could lead to readmission (3). They reported that the incidence of postoperative complications was $48 \%$, but the study did not analyze detailed types of postoperative complications. Various kinds of complications can occur after esophagectomy, and the incidence of complications has been reported up to $50 \%$ (9). We hypothesized that specific but not all complications might be related to readmission.

Our initial hypothesis was that postoperative vocal cord palsy can cause swallowing difficulties, aspiration pneumonia, and related readmission. Our group has an aggressive surgical policy for mediastinal lymphadenectomy, which can be associated with postoperative vocal cord palsy, in approximately $20 \%$ of cases. Postoperative vocal cord palsy can cause swallowing difficulties, poor oral intake, and poor quality of life during the follow-up period (10). Baba et al. reported that patients with severe hoarseness and permanent recurrent nerve paralysis showed poor food intake at 24 months or less postoperatively and restricted daily activity and difficulty in talking at 60 months or more after the operation (10). In addition, several papers reported that recurrent laryngeal nerve palsy is related to postoperative pulmonary complications due to recurrent laryngeal nerve palsy-related aspiration (11). Therefore, we hypothesized that recurrent laryngeal nerve palsyrelated aspiration could affect the postoperative course after discharge and result in readmission. In contrast to our initial hypothesis, the data showed that postoperative vocal cord palsy was not related to readmission after esophagectomy. This result might be related to the intensive patient education after operation. We administered an education program on postoperative care after esophagectomy, which specifically focused on diet and ways to eat food in patients with vocal cord palsy and difficulty swallowing.

In contrast to the initial hypothesis, postoperative anastomotic leakage was found to be related to readmission. Twelve (30.7\%) patients required endoscopic ballooning due to the anastomotic stricture which might be related to the postoperative anastomotic leakage, and 4 among the 7 patients with wound problems had problems with neck wounds. Neck wound problems could be related to subclinical minor anastomotic leakage. Therefore, the 16 (41\%) patients that experienced this could have associated anastomotic problems. The incidence of anastomotic leakage after initial operation in this study was $10.3 \%$, which was comparable and slightly lower than previous reports, especially the results of the Japan Nationwide database (rate of anastomotic leakage; 13.3\%) (9). Our incidence of anastomotic leakage was acceptable, but the incidence of anastomotic leakage should be decreased to reduce readmission rates. Therefore, risk factors of anastomotic leakage identified in our series will be analyzed in further studies. Based on these results, technical modification for anastomosis and conduit preparation and improvement of perioperative management should also be studied.

Even though the incidence was low, there were several readmissions related to jejunostomy. We placed the jejunostomy tube for proper nutritional support during the perioperative period. This approach was used particularly when patients are expected to vocal cord palsy due to aggressive mediastinal lymph node dissection; therefore, enteral nutritional support instead of oral intake could be helpful. In some cases, the jejunostomy tube can cause complications, such as intestinal obstruction; in our series, two patients suffered from mechanical obstruction attributable to the jejunostomy tube. Therefore, alternative routes for postoperative nutrition instead of jejunostomy and surgical technique modifications to reduce jejunostomyrelated complications should be studied further. Ninomiya et al. suggested that inserting the catheter for gastrostomy through a gastric conduit could prevent jejunostomy-related bowel obstruction (12).

In previous studies, venous thromboembolism was a main reason for readmission, and prophylaxis for venous thromboembolism was recommended (3). However, the results of this study did not indicate any thrombosis-related readmission. A systematic review of the incidence of venous thromboembolism in Asian patients indicated that population-wide incidence estimates in Asia were approximately $15 \%$ to $20 \%$ of the levels recorded in Western countries (13). In addition, venous thromboembolism is rarely reported during immediate postoperative periods for initial esophagectomy. It is possible that the low incidence of postoperative thrombotic events in our patients could be related to ethnic differences and differences between Western and Eastern patients. Therefore, the cause of readmission after esophagectomy could different depending on race, region, and country. 
Based on this, postoperative care and plans to reduce readmission rates should be individualized based on race and geographical region.

There were several limitations to this study. First, this study analyzed patients from a single institution over 10 years and the pathologies were mainly esophageal squamous cell carcinoma. Therefore, the results have to be interpreted cautiously because each institution and surgeons have different surgical polices, and frequent complications could differ. In addition, similar to rates of venous thromboembolism, there were racial differences. However, these results reflect cases that were treated at our institution and can provide guidance for improvements in patient treatment and procedural approaches. Based on the results, consideration of complications and readmission should be individualized to optimize patient outcomes. Second, due to the characteristics of the National Health Insurance of Korea, the rate of readmission might be overestimated. Specifically, the $4(10.2 \%)$ patients that were readmitted due to poor oral intake could be related to the he characteristics of the National Health Insurance. In the National Health Insurance of Korea system, the patients pay only about 10 US dollars for hospitalization per day, which is relatively inexpensive compared to other countries, especially the United States. Due to the low hospitalization costs, patients expect and demand hospitalization even for mild symptoms that can be managed in outpatient clinics. Finally, within the same context, the economic burden due to readmission referred to in this paper might be smaller than in other countries.

In conclusion, the rate of readmission within 30 days after esophagectomy was $13.4 \%$ in our data and was primarily related to postoperative anastomotic leakage and wound problems. Based on the results, technical improvements to reduce anastomotic leakage and wound problems are necessary to improve esophagectomy surgical quality and patient outcomes.

\section{Acknowledgments}

Funding: This research was supported by Basic Science Research Program through the National Research Foundation of Korea (NRF) funded by the Ministry of Education (2019R1I1A1A01055513).

\section{Footnote}

Conflicts of Interest: The authors have no conflicts of interest to declare.
Ethical Statement: The authors are accountable for all aspects of the work in ensuring that questions related to the accuracy or integrity of any part of the work are appropriately investigated and resolved. This retrospective study was approved by the institutional review board of our hospital (4-2018-0580).

\section{References}

1. Hu Y, McMurry TL, Stukenborg GJ, et al. Readmission predicts 90-day mortality after esophagectomy: Analysis of Surveillance, Epidemiology, and End Results Registry linked to Medicare outcomes. J Thorac Cardiovasc Surg 2015;150:1254-60.

2. Bailey SH, Bull DA, Harpole DH, et al. Outcomes after esophagectomy: a ten-year prospective cohort. Ann Thorac Surg 2003;75:217-22; discussion 222.

3. Shah SP, Xu T, Hooker CM, et al. Why are patients being readmitted after surgery for esophageal cancer? J Thorac Cardiovasc Surg 2015;149:1384-9; discussion 1389-91.

4. Fernandez FG, Khullar O, Force SD, et al. Hospital readmission is associated with poor survival after esophagectomy for esophageal cancer. Ann Thorac Surg 2015;99:292-7.

5. Low DE, Alderson D, Cecconello I, et al. International Consensus on Standardization of Data Collection for Complications Associated With Esophagectomy: Esophagectomy Complications Consensus Group (ECCG). Ann Surg 2015;262:286-94.

6. Clavien PA, Barkun J, de Oliveira ML, et al. The ClavienDindo classification of surgical complications: five-year experience. Ann Surg 2009;250:187-96.

7. Rice TW, Ishwaran H, Hofstetter WL, et al. Recommendations for pathologic staging (pTNM) of cancer of the esophagus and esophagogastric junction for the 8th edition AJCC/UICC staging manuals. Dis Esophagus 2016;29:897-905.

8. Sundaram A, Srinivasan A, Baker S, et al. Readmission and risk factors for readmission following esophagectomy for esophageal cancer. J Gastrointest Surg 2015;19:581-5; discussion 586.

9. Takeuchi H, Miyata H, Gotoh M, et al. A risk model for esophagectomy using data of 5354 patients included in a Japanese nationwide web-based database. Ann Surg 2014;260:259-66.

10. Baba M, Aikou T, Natsugoe S, et al. Quality of life following esophagectomy with three-field 
lymphadenectomy for carcinoma, focusing on its relationship to vocal cord palsy. Dis Esophagus 2017;11:28-34.

11. Atkins BZ, D'Amico TA. Respiratory complications after esophagectomy. Thorac Surg Clin 2006;16:35-48, vi.

12. Ninomiya I, Makino I, Fujimura T, et al. Feeding

Cite this article as: Park SY, Kim DJ, Byun GE. Incidence and risk factors of readmission after esophagectomy for esophageal cancer. J Thorac Dis 2019;11(11):4700-4707. doi: 10.21037/ jtd.2019.10.34 catheter gastrostomy at esophagectomy with gastric tube reconstruction through posterior mediastinal route. Esophagus 2011;8:217-23.

13. Lee LH, Gallus A, Jindal R, et al. Incidence of Venous Thromboembolism in Asian Populations: A Systematic Review. Thromb Haemost 2017;117:2243-60. 